\title{
Mitral regurgitation surgery in patients with ischemic cardiomyopathy and ischemic mitral regurgitation: Factors that influence survival
}

\author{
Simon Maltais, MD, PhD, ${ }^{a}$ Hartzell V. Schaff, MD, ${ }^{a}$ Richard C. Daly, MD, ${ }^{a}$ Rakesh M. Suri, MD, PhD, \\ Joseph A. Dearani, MD, ${ }^{\mathrm{a}}$ Thoralf M. Sundt III, MD, ${ }^{\mathrm{a}}$ Maurice Enriquez-Sarano, MD, ${ }^{\mathrm{b}}$ \\ Yan Topilsky, MD, ${ }^{\mathrm{b}}$ and Soon J. Park, MD, MSc ${ }^{\mathrm{a}}$
}

\begin{abstract}
Objective: The treatment of patients with ischemic cardiomyopathy and concomitant mitral regurgitation can be challenging and is associated with reduced long-term survival. It is unclear how mitral valve repair versus replacement affects subsequent outcome. Therefore, we conducted this study to understand the predictors of mortality and to delineate the role of mitral valve repair versus replacement in this high-risk population.
\end{abstract}

\begin{abstract}
Methods: From 1993 to 2007, 431 patients (mean age, $70 \pm 9$ years) with ischemic cardiomyopathy (left ventricular ejection fraction $\leq 45 \%$ ) and significant ischemic mitral regurgitation $(>2)$ were identified. Patients (44) with concomitant mitral stenosis were excluded from the analysis. A homogeneous group of 387 patients underwent combined coronary artery bypass grafting and mitral valve surgery, mitral valve repair in $302(78 \%)$ and mitral valve replacement in $85(22 \%)$. Uni- and multivariate analyses were performed on the entire cohort, and the predictors of mortality were identified in 2 distinct risk phases. Furthermore, we specifically examined the impact of mitral valve repair versus replacement by comparing 2 propensitymatched subgroups.
\end{abstract}

Results: Follow-up was 100\% complete (median, 3.6 years; range, $0-15$ years). Overall 1-, 5-, and 10-year survivals were $82.7 \%, 55.2 \%$, and $24.3 \%$, respectively, for the entire group. The risk factors for an increased mortality within the first year of surgery included previous coronary artery bypass grafting (hazard ratio $=3.39$; $P<.001$ ), emergency/urgent status (hazard ratio $=2.08 ; P=.007$ ), age (hazard ratio $=1.5 ; P=.03$ ), and low left ventricular ejection fraction (hazard ratio $=1.31 ; P=.026$ ). Thereafter, only age (hazard ratio $=1.58 ; P<.001$ ), diabetes (hazard ratio $=2.5 ; P=.001$ ), and preoperative renal insufficiency (hazard ratio $=1.72 ; P=.025$ ) were predictive. The status of mitral valve repair versus replacement did not influence survival, and this was confirmed by comparable survival in propensity-matched analyses.

Conclusions: Survival after combined coronary artery bypass grafting and mitral valve surgery in patients with ischemic cardiomyopathy (left ventricular ejection fraction $\leq 45 \%$ ) and mitral regurgitation is compromised and mostly influenced by factors related to the patient's condition at the time of surgery. The specifics of mitral valve repair versus replacement did not seem to affect survival. (J Thorac Cardiovasc Surg 2011;142:995-1001)

Earn CME credits at

http://cme.ctsnetjournals.org

Patients with ischemic heart disease and mitral regurgitation (MR) comprise one of the more perplexing and

From the Divisions of Cardiovascular Surgery ${ }^{\mathrm{a}}$ and Cardiovascular Medicine, ${ }^{\mathrm{b}}$ Mayo Clinic College of Medicine, Rochester, Minn.

Disclosures: Authors have nothing to disclose with regard to commercial support.

Read at the 91st Annual Meeting of The American Association for Thoracic Surgery, Philadelphia, Pennsylvania, May 7-11, 2011.

Received for publication April 8, 2011; revisions received June 21, 2011; accepted for publication July 20, 2011; available ahead of print Aug 22, 2011.

Address for reprints: Soon J. Park, MD, MSc, Division of Cardiovascular Surgery, Mayo Clinic College of Medicine, 200 First Street SW, Rochester, MN 55905 (E-mail: Park.Soon@mayo.edu).

$0022-5223 / \$ 36.00$

Copyright (c) 2011 by The American Association for Thoracic Surgery doi:10.1016/j.jtcvs.2011.07.044 challenging groups to treat in cardiac surgery. Some of these patients have 2 independent disease processes of coronary ischemia and myxomatous MR, and they tend to have a favorable survival outcome after surgical correction. ${ }^{1-3}$ However, the outcome seems to be more guarded in those who have coronary ischemia and functional MR, that is, ischemic MR (IMR). ${ }^{4}$ In this population, IMR can be due to various pathophysiologic processes ranging from acute papillary muscle ischemia/rupture to chronic left ventricular (LV) remodeling after myocardial infarction, resulting in tethered and incompetent mitral leaflets unable to coapt. Accordingly, their clinical presentation varies, ranging from acute pulmonary edema to recurrent bouts of congestive heart failure. This has been shown to influence the surgeon's decision to perform mitral valve repair (MVP) or mitral valve replacement (MVR) ${ }^{5,6}$ All these factors contribute to the difficulty in understanding the comparative efficacy of MVP versus MVR, and it is not surprising to have 


$$
\begin{aligned}
& \text { Abbreviations and Acronyms } \\
& \text { CABG }=\text { coronary artery bypass grafting } \\
& \text { HR }=\text { hazard ratio } \\
& \text { ICM }=\text { ischemic cardiomyopathy } \\
& \text { IMR }=\text { ischemic mitral regurgitation } \\
& \text { LAD }=\text { left anterior descending } \\
& \text { LITA }=\text { left internal thoracic artery } \\
& \text { LV }=\text { left ventricular, left ventricle } \\
& \text { LVEF }=\text { left ventricular ejection fraction } \\
& \text { MR }=\text { mitral regurgitation } \\
& \text { MV }=\text { mitral valve } \\
& \text { MVP }=\text { mitral valve repair } \\
& \text { MVR }=\text { mitral valve replacement }
\end{aligned}
$$

MVP and MVR, propensity matched on 14 baseline characteristic variables (age, gender, hypertension, diabetes, history of smoking, body surface area, preoperative New York Heart Association class, chronic renal failure, preoperative dialysis, previous CABG surgery, previous valve procedure, history of congestive heart failure, LVEF, and emergency/urgent status of the procedure).

\section{Definitions}

The cause of MR was presumed to be ischemic. All patients had a significant degree of mitral annular dilation and LV dysfunction due to prior myocardial infarction. In patients who required more complex repair for mixed valvular pathology, operative and echocardiography reports were reviewed to confirm that myocardial ischemia was the primary mechanism for MR. The operative and echocardiographic findings were reviewed in detail in these patients, and they were deemed to have IMR on the basis of leaflet tethering, prior myocardial infarction, and leaflet tethering. All patients included in this study had an undersized ring/band implanted when applicable. In patients with repairs, the ring was chosen according to the undersized intercommissural distance when applicable. In patients with a $63-$ to $65-\mathrm{mm}$ posterior band, the band was cut and undersized to the appropriate length. The exact nature of chordal preservation in each patient undergoing MVR was not established. However, our institutional policy has been to preserve the posterior leaflet whenever possible with an increasing recent tendency toward preserving as much of the anterior leaflet and by transposing it to the posterior annulus.

\section{Follow-up}

Patients were followed systematically by using mailed questionnaires, telephone interview, or examination at the Mayo Clinic. Clinical followup for both patients with MVR and MVP was $100 \%$ complete. Mean follow-up among survivors was 4.2 years (range, $0-15.7$ years).

\section{Statistical Analysis}

Descriptive statistics for categoric variables are reported as frequency and percentage, and continuous variables are reported as mean (standard deviation) or median (range) as appropriate. Categoric variables were compared between MVP and MVR groups using the chi-square test, and continuous variables were compared using 2-sample $t$ test or Wilcoxon rank-sum test when appropriate. The Kaplan-Meier method was used to draw survival curves and calculate 1-, 5-, and 10-year survival statistics. Cox regression models were used to find the univariate and multivariate predictors of early (1-year) and long-term (late or constant) survivals. The multivariable model considered univariate significant variables $(P<.05)$ with model selection using the stepwise method (backward and forward methods resulted in the same model). All statistical tests were 2-sided with the alpha level set at .05 for statistical significance.

\section{RESULTS \\ Patient Characteristics}

From 1993 to 2007, 431 patients were identified and required a combined CABG and MVP/MVR procedure. Of these, 44 patients were excluded because they had mitral stenosis, yielding 387 patients for this study. The mean age at the time of surgery was $70.1 \pm 9.1$ years (range 43-91 years), and 261 patients were male (67\%). CABG surgery was performed in all patients. MVP was performed in 302 patients $(78 \%)$, and MVR was performed in 85 patients $(22 \%)$. All patients had LV dysfunction (LVEF $\leq$ $45 \%$ ), and the mean LVEF by preoperative transthoracic echocardiography was $33.6 \% \pm 8.4 \%$ (range, 9-45). 
TABLE 1. Patient characteristics

\begin{tabular}{|c|c|c|c|c|}
\hline Risk factors & All groups, $\mathbf{n}=\mathbf{3 8 7}$ & Repair, $\mathbf{n}=302$ & Replacement, $\mathrm{n}=\mathbf{8 5}$ & $P$ value \\
\hline Age (y) & $70.1 \pm 9.1$ & $70.3 \pm 8.8$ & $69.5 \pm 9.9$ & .93 \\
\hline Female $(\mathrm{n}, \%)$ & $126(33,1)$ & $95(31.5)$ & $31(36.5)$ & .38 \\
\hline Hypertension (n, \%) & $273(70.5)$ & $215(71.2)$ & $58(68.2)$ & .59 \\
\hline Diabetes $(\mathrm{n}, \%)$ & $126(32.5)$ & $104(34.4)$ & $22(25.9)$ & .14 \\
\hline History of smoking $(\mathrm{n}, \%)$ & $252(65.1)$ & $200(66.2)$ & $52(62.2$ & .22 \\
\hline $\operatorname{BSA}\left(\mathrm{m}^{2}\right)$ & $1.9 \pm 0.2$ & $1.9 \pm 0.2$ & $1.9 \pm 0.2$ & .31 \\
\hline NYHA class III or IV (n, \%) & $335(86.6)$ & $257(85.2)$ & $78(91.3)$ & .11 \\
\hline Chronic renal failure $(\mathrm{n}, \%)$ & $39(10.1)$ & $31(10.3)$ & $8(9.4)$ & .82 \\
\hline Preoperative creatinine $(\mathrm{mg} / \mathrm{dL})$ & $1.6 \pm 0.9$ & $1.6 \pm 1.1$ & $1.5 \pm 0.7$ & .68 \\
\hline Preoperative dialysis $(\mathrm{n}, \%)$ & $7(1.8)$ & $5(1.6)$ & $2(2.4)$ & .67 \\
\hline Previous CABG surgery $(\mathrm{n}, \%)$ & $51(13.2)$ & $28(9.3)$ & $23(27.1)$ & $<.0001$ \\
\hline Previous mitral procedure $(\mathrm{n}, \%)$ & $23(5.9)$ & $8(2.6)$ & 15 (17.6) & $<.0001$ \\
\hline History of CHF (n, \%) & $226(58.4)$ & $178(58.9)$ & $48(56.5)$ & .68 \\
\hline $\operatorname{LVEF}(\%)$ & $33.6 \pm 8.4$ & $33.6 \pm 8.5$ & $34.3 \pm 7.9$ & .50 \\
\hline Emergency/urgent operation (n, \%) & $13(3.4)$ & $10(3.3)$ & $3(3.5)$ & .96 \\
\hline
\end{tabular}

BSA, Body surface area; $C H F$, congestive heart failure; NYHA, New York Heart Association.

Baseline characteristics of all patients and 2 subgroups according to the MV procedure (repair vs replacement) are presented in Table 1. The patients in the MVR group had a significantly higher incidence of CABG $(27.1 \%$ vs $9.3 \% ; P<.0001)$ or MV surgery $(17.6 \%$ vs $2.6 \%$; $P<.0001)$ in the past.

\section{Operative Details}

Table 2 summarizes the operative details for all patients included in this study. All surgeries were performed through a median sternotomy using cardiopulmonary bypass, blood cardioplegia, and the standard aortic crossclamping operative technique. The LITA was used in

TABLE 2. Operative details

\begin{tabular}{lc}
\hline \multicolumn{1}{c}{ Procedure details } & All groups, $\mathbf{n}=\mathbf{3 8 7}$ \\
\hline Mitral repair ( $\mathrm{n}=302$ patient) & \\
$\quad$ Annuloplasty (n, \%) & $302(100)$ \\
Concomitant leaflet resection (n, \%) & $31(8)$ \\
Ring/band sizes (mm) & \\
$\quad 24-28$ & $128(42)$ \\
$30-34$ & $109(36)$ \\
63-65 & $69(22)$ \\
Mitral replacement (n=85 patients) & \\
Bioprosthesis (n, \%) & $46(12)$ \\
Mechanical (n, \%) & $39(10)$ \\
LITA use (n, \%) & $290(75)$ \\
No. of distal anatomosis (n, \%) & \\
1 & $109(28)$ \\
2-4 & $262(68)$ \\
$>4$ & $16(4)$ \\
Concomitant valve procedures & \\
Aortic valve replacement & $60(15)$ \\
Tricuspid valve repair/replacement & $41(11)$ \\
Cardiopulmonary bypass time (min) & $124.4 \pm 47.5$ \\
Aortic crossclamping time (min) & $82.2 \pm 31.6$ \\
\hline
\end{tabular}

290 patients $(75 \%)$. MVP comprised implanting a posterior annuloplasty ring/band only in 279 patients $(92 \%)$. Thirty-one patients $(8 \%)$ required a more complex repair for mixed valvular lesions. In patients undergoing MVR, $46(54 \%)$ received a bioprosthetic valve and $39(46 \%)$ received a mechanical valve. The mean cardiopulmonary bypass time was $124.4 \pm 47.5$ minutes (range, 30-340), and the mean aortic crossclamp time was $82.2 \pm 31.6 \mathrm{~min}$ utes (range, 16.5-46.4). The number of treated patients per year was consistent throughout the study period, and the survival was not affected by the surgical era (data not shown; $P=.69$ ).

\section{Overall Survival Analysis}

The observed in-hospital or 30-day mortality was 5\% (21 patients). The overall reported 1-, 5-, and 10-year survivals for the entire cohort were $84.0 \%, 55.4 \%$, and $25.4 \%$, respectively (Figure 1). The Kaplan-Meier survival curve had 2 different mortality risk phases: an acute phase

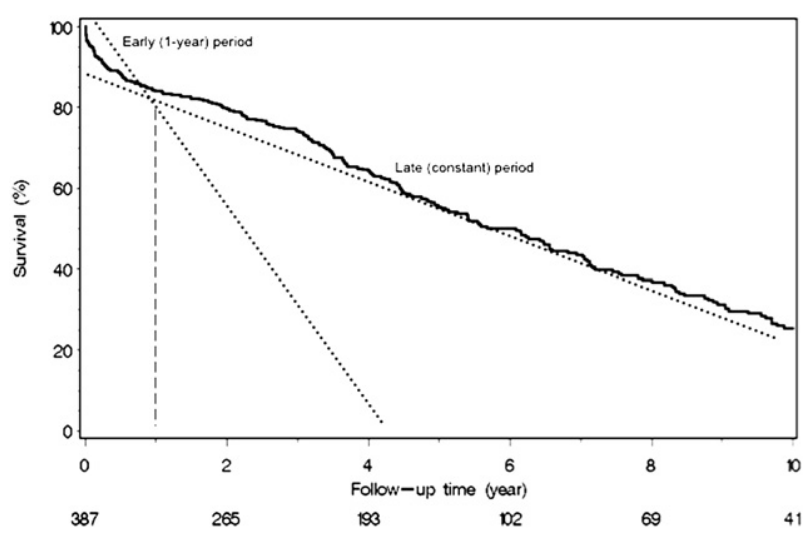

FIGURE 1. Overall reported 1-, 5-, and 10-year survivals for the entire cohort were $82.7 \%, 55.2 \%$, and $24.3 \%$, respectively. 
TABLE 3. Multivariate risk factor analysis for death in patients undergoing concomitant coronary artery bypass grafting and mitral valve surgery

\begin{tabular}{llll}
\hline & $\begin{array}{c}\text { Early }(\mathbf{1}-\mathbf{y}) \\
\text { hazard }\end{array}$ & & $\begin{array}{c}\text { Late } \\
\text { (constant hazard })\end{array}$ \\
\cline { 2 - 2 } Risk factors & HR $(\boldsymbol{P}$ value $)$ & & HR $(\boldsymbol{P}$ value $)$ \\
\hline Previous CABG surgery & $3.39(<.001)$ & & \\
Emergency/urgent status & $2.08(.007)$ & & \\
LVEF (per 10\% decrease) & $1.31(.02)$ & & \\
Age & $1.50(.026)$ & & $1.58(<.001)$ \\
Renal insufficiency & & $1.72(.025)$ \\
Diabetes & & $2.50(<.001)$ \\
\hline
\end{tabular}

Multivariate Cox regression analysis model for early (1-year) and late (constant) survivals.

corresponding to the early (1-year) period and a second late (constant) phase.

\section{Predictors of Early (1-Year) and Late (Constant) Mortality}

Table 3 summarizes identified risk factors based on multivariate Cox's regression analyses. Previous CABG surgery (hazard ratio $[\mathrm{HR}]=3.39 ; P<.001$ ), emergency/urgent status of the procedure $(\mathrm{HR}=2.08 ; P=.007)$, age $(\mathrm{HR}=1.5$; $P=.03)$, and low $\operatorname{LVEF}(\mathrm{HR}=1.31 ; P=.026)$ were predictive of early mortality. The factors found to influence the late (constant) mortality were age ( $\mathrm{HR}=1.58 ; P<.001)$, diabetes $(\mathrm{HR}=2.5 ; P=.001)$, and preoperative renal insufficiency $(\mathrm{HR}=1.72 ; P=.025)$. The type of MV procedure performed (MVP or MVR) did not influence early or late mortality.

\section{Propensity-Matched Comparisons Comparing Mitral Valve Procedures}

We noted comparable survival between 2 propensitymatched subgroups composed of 76 patients in each type of MV procedure. There were no significant differences between the MVP and MVR groups; 1-year $(81.9 \%$ vs $79.6 \% ; P=.72)$ and 5 -year $(44.0 \%$ vs $54.2 \% ; P=.72)$ survivals were comparable.

\section{DISCUSSION}

The resurgence of interest in MVP over the past decade has provided long-term data supporting the superiority of repair over replacement in patients with normal $\mathrm{LV}$ function or degenerative/myxomatous valve disease. ${ }^{11}$ With valverelated advantages aside, it is generally accepted that in this population, MVP has demonstrated improvements over MVR in terms of heart function recovery and longterm survival. ${ }^{12}$ However, in patients with ICM and IMR, the impact of MVP over MVR remains controversial. Few retrospective studies have focused on the predictors of survival in patients with severe IMR and ICM. ${ }^{7,8,13}$ The present study included patients who underwent a combined CABG and MVP/MVR for significant ICM (LVEF $\leq 45 \%)$ and significant IMR over the past 15 years.

\section{Key Findings}

The survival curve of our entire cohort demonstrated 2 different mortality risk phases as shown in Figure 1. Accordingly, we divided the mortality risk analyses into the early phase (up to 1 year after surgery) and the late phase (beyond the first year). This is concordant with previously published studies by Gillinov and associates ${ }^{7}$ describing distinct phases of instantaneous risk of death in these patients. In our study, factors influencing mortality in the early phase included a history of CABG surgery $(\mathrm{HR}=3.39$; $P<.001)$, emergency/urgent status of the surgery $(\mathrm{HR}=2.08 ; P=.007)$, age $(\mathrm{HR}=1.5 ; P=.03)$, and low LVEF $(\mathrm{HR}=1.31 ; P=.026)$, as shown in Table 3 . These risk factors were reflective of the associated technical challenges of a redo CABG surgery, patient's medical status requiring nonelective surgery, and poor cardiac reserve. The finding of these risk factors is consistent with our clinical impression that an expected mortality within the first year would be high, for example, in a patient with an LVEF of $30 \%$ who would require a technically challenging redo CABG and MVP/MVR on an emergency/urgent basis. ${ }^{4}$ For this patient, the expected operative mortality (30-day or in-hospital) also would be high, but we were unable to perform meaningful multivariate analyses for this end point, because the operative mortality observed in this study was low $(5 \%)$.

Once patients survived the first year, their subsequent mortality was mostly based on their noncardiac comorbidities of diabetes $(\mathrm{HR}=2.5 ; P=.001)$, renal insufficiency $(\mathrm{HR}=1.72 ; P=.025)$, and age $(\mathrm{HR}=1.58 ; P<.001)$. One could speculate many potential reasons for the finding that none of the cardiac specific risk factors played a role on survival during the late phase. One of the major reasons may be that because all patients in the study started with poor cardiac reserve (LVEF $\leq 45 \%$ with significant MR) before surgery, such significant underlying cardiac dysfunction had a uniform and overwhelming influence on survival. The specifics of mitral surgery (MVP vs MVR) had no influence on survival in the early or late risk phase in this study cohort. Furthermore, in small subgroup analysis, we confirmed that the type of repair (rigid/flexible, complete/ partial, concomitant leaflet procedure) did not influence survival in patients with repairs $(P=.24)$.

We examined the issue of MVP versus MVR in this specific population by comparing 2 propensity subgroups. We identified a matching pair of patients undergoing MVP for 76 of 85 patients undergoing MVR on the basis of 14 important clinical variables, as mentioned in the "Materials and Methods" section. As shown in Figure 2, survival in these patients with an LVEF $45 \%$ or less was comparable between patients with MVR and MVP. This is comparable 


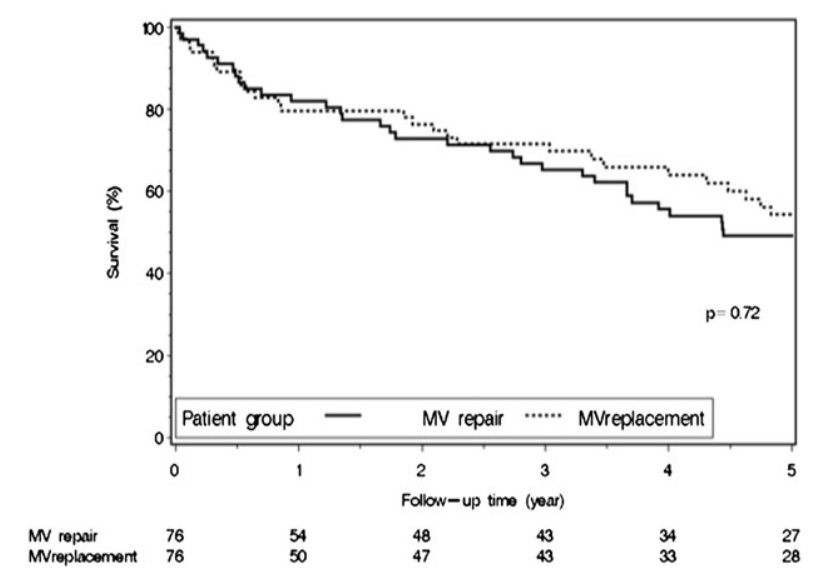

FIGURE 2. Survival results for propensity-matched patients. There were no differences between patients undergoing MVR or MVP. Survival was comparable between propensity-matched groups of patients $(P=.72)$. $M V$, Mitral valve.

to previously published studies suggesting that survival is limited after MV operation and CABG, with no advantage of either strategy for managing the MV. ${ }^{4,7,14}$ Gillinov and associates $^{7}$ also attempted to address the question of MVP versus MVR. Their surgical practice of MVP versus MVR was significantly confounded with the patient's condition, and their matched pair yielded "only one end of spectrum of IMR (the most complex with the sickest patients)." They exercised sophisticated and elaborate statistical analyses to compare different quintile risk subgroups based on propensity-adjusted for MVP versus MVR selection factors. The experience reported by Grossi and associates ${ }^{8}$ further demonstrated a high degree of confounding relationship between the type of MV procedure and the preoperative patient characteristics of angina and New York Heart Association class. In our cohort, we also noted some differences in baseline characteristics between the MVP and MVR groups, but such differences were only related to prior cardiac surgery (Table 1). By selecting a relatively homogeneous study population with an LVEF of $45 \%$ or less within the recent surgical era, we were able to compare a meaningful number of propensity-matched patients and examine the survival impact of MVP versus MVR specifically.

\section{Other Important Considerations}

Several groups have shown that survival after coronary revascularization is strongly influenced by the use of the left internal thoracic artery (LITA) to the left anterior descending (LAD) artery. ${ }^{15,16}$ In this study, $75 \%$ of patients with an MV procedure and concomitant $\mathrm{CABG}$ had the LITA to the LAD used. We strongly believe that previously reported differences in survival observed in MVP compared with MVR in patients with ICM may be related to the low incidence of using the LITA to the LAD, especially in patients with MVR $(15 \%) .{ }^{7}$ When there is an associated LAD pathology, the LITA should always be used in this population with ischemic LV function to optimize LV recovery and possibly survival. Furthermore, in patients with MVP, residual MR can be present and negatively influence the degree of LV recovery and remodeling. In a recent study performed in 111 patients undergoing MV surgery, De Bonis and colleagues ${ }^{9}$ showed that the progression of LV remodeling paralleled the recurrence of MR. At 3 years, freedom from recurrence of MR of $2+$ or greater was $74 \%$ in those who showed a reverse remodeling process. In a multicenter study, Shiota and associates ${ }^{10}$ reported a surprisingly high $33 \%$ recurrence of MR 6 months after mitral annuloplasty in patients with ICM. In their study, recurrent MR was associated with increased LV size and decreased LVEF. Hypothetically, in terms of recurrence of MR, MVR could represent a more reproducible and durable surgical treatment in these patients. Of course, preservation of as much MV apparatus as possible at the time of MVR would be important. ${ }^{17,18}$

\section{Limitations}

One of the major limitations of our study is that it is a retrospective review of patients who underwent a combined surgery of CABG and MVP or MVR, in which a selection bias plays a role, and it is hard to account for all of its impact on outcome. We also do not have a comparison group with no concomitant MV procedures. Therefore, the added benefit of MVP/MVR is still speculative on the presumption that reducing MR renders a therapeutic benefit on outcome. The proposed National Institutes of Health-sponsored cardiac surgery network studies of prospective randomized trials involving patients with IMR would be critically important in providing further answers in treating these perplexing and challenging patients. The end points of this study were predictors of death, and we did not analyze other complications because we have already described valve procedurerelated differences in durability in the literature. ${ }^{19}$

\section{CONCLUSIONS}

We evaluated the predictors of survival in patients with ICM and IMR. We identified 2 distinct risk phases based on the survival curve kinetics. The risk predictors of mortality during the early phase (1-year) were factors associated with the patient's presenting condition and projected technical difficulty at the time of the intervention (previous CABG surgery, emergency intervention, low LVEF). In the late (after the first year) phase, patients' comorbidities (age, diabetes, and renal insufficiency) were the determinants of mortality. In patients with ICM and IMR, the nature of the MV intervention (MVP or MVR) had no influence on survival.

We would like express our gratitude to Judy Lenoch and Zhuo $\mathrm{Li}$ for their help with the database and statistical analyses. 


\section{References}

1. Kuwaki K, Kiyofumi M, Tsukamoto M, Abe T. Early and late results of mitral valve repair for mitral valve regurgitation. Significant risk factors of reoperation. J Cardiovasc Surg (Torino). 2000;41:187-92.

2. Enriquez-Sarano M, Schaff HV, Orszulak TA, Tajik AJ, Bailey KR, Frye RL. Valve repair improves the outcome of surgery for mitral regurgitation. A multivariate analysis. Circulation. 1995;91:1022-8.

3. Ling LH, Enriquez-Sarano M, Seward JB, et al. Clinical outcome of mitral regurgitation due to flail leaflet. $N$ Engl J Med. 1996;335:1417-23.

4. Dahlberg PS, Orszulak TA, Mullany CJ, Daly RC, Enriquez-Sarano M, Schaff HV. Late outcome of mitral valve surgery for patients with coronary artery disease. Ann Thorac Surg. 2003;76:1539-487; discussion 1547-8.

5. Orszulak TA, Schaff HV, Danielson GK, et al. Mitral regurgitation due to ruptured chordae tendineae. Early and late results of valve repair. J Thorac Cardiovasc Surg. 1985;89:491-8.

6. Tribouilloy CM, Enriquez-Sarano M, Schaff HV, et al. Impact of preoperative symptoms on survival after surgical correction of organic mitral regurgitation: rationale for optimizing surgical indications. Circulation. 1999;99: 400-5.

7. Gillinov AM, Faber C, Houghtaling PL, et al. Repair versus replacement for degenerative mitral valve disease with coexisting ischemic heart disease. J Thorac Cardiovasc Surg. 2003;125:1350-62.

8. Grossi EA, Goldberg JD, LaPietra A, et al. Ischemic mitral valve reconstruction and replacement: comparison of long-term survival and complications. J Thorac Cardiovasc Surg. 2001;122:1107-24.

9. De Bonis M, Lapenna E, Verzini A, et al. Recurrence of mitral regurgitation parallels the absence of left ventricular reverse remodeling after mitral repair in advanced dilated cardiomyopathy. Ann Thorac Surg. 2008;85:932-9.

10. Shiota M, Gillinov AM, Takasaki K, Fukuda S, Shiota T. Recurrent mitral regurgitation late after annuloplasty for ischemic mitral regurgitation. Echocardiography. 2011;28:161-6.

11. Lawrie GM. Mitral valve repair vs replacement. Current recommendations and long-term results. Cardiol Clin. 1998;16:437-48.

12. Nishimura RA, Carabello BA, Faxon DP, et al. ACC/AHA 2008 Guideline update on valvular heart disease: focused update on infective endocarditis: a report of the American College of Cardiology/American Heart Association Task Force on Practice Guidelines endorsed by the Society of Cardiovascular Anesthesiologists, Society for Cardiovascular Angiography and Interventions, and Society of Thoracic Surgeons. J Am Coll Cardiol. 2008;52:676-85.

13. Cohn LH, Rizzo RJ, Adams DH, et al. The effect of pathophysiology on the surgical treatment of ischemic mitral regurgitation: operative and late risks of repair versus replacement. Eur J Cardiothorac Surg. 1995;9:568-74.

14. Lee EM, Shapiro LM, Wells FC. Superiority of mitral valve repair in surgery for degenerative mitral regurgitation. Eur Heart J. 1997;18:655-63.

15. Pick AW, Orszulak TA, Anderson BJ, Schaff HV. Single versus bilateral internal mammary artery grafts: 10-year outcome analysis. Ann Thorac Surg. 1997;64: 599-605.

16. Boylan MJ, Lytle BW, Loop FD, et al. Surgical treatment of isolated left anterior descending coronary stenosis. Comparison of left internal mammary artery and venous autograft at 18 to 20 years of follow-up. J Thorac Cardiovasc Surg. 1994;107:657-62.

17. Borger MA, Yau TM, Rao V, Scully HE, David TE. Reoperative mitral valve replacement: importance of preservation of the subvalvular apparatus. Ann Thorac Surg. 2002;74:1482-7.

18. Okita Y, Miki S, Ueda Y, Tahata T, Sakai T. Left ventricular function after mitral valve replacement with or without chordal preservation. J Heart Valve Dis. 1995; 4(Suppl 2):S181-93.

19. Mohty D, Orszulak TA, Schaff HV, Avierinos JF, Tajik JA, Enriquez-Sarano M. Very long-term survival and durability of mitral valve repair for mitral valve prolapse. Circulation. 2001;104(12 Suppl. 1):I1-7.

\section{Discussion}

Dr Patrick McCarthy (Chicago, Ill). By disclosure, I am a coinventor of the IMR ETlogix ring (Edwards Lifesciences, Irvine, Calif).

Congratulations, Dr Maltais, on an excellent and timely presentation. We look forward to the results of the ongoing National
Institutes of Health prospective randomized trial that will give further information on this important subject.

As you note, patient factors were important to survival, but we can't modify those. As surgeons, we can decide, however, between MVR and MVP. So I have questions about how that decision was made and late outcomes.

First, can you provide insight into why MVR was sometimes chosen? Was it influenced by the degree of leaflet tethering or other factors seen on echocardiography? Was MVR used for worse ventricles or more complex and extensive MR? Others have found no difference in survival between MVP and MVR in single-center studies, but did you look at other outcomes, such as need for transplant or ventricular assist device, late New York Heart functional class, and rehospitalization for cardiac cause, and do you have any data on late MR recurrence? Finally, after this study, what do you do now, repair or replace?

Dr Maltais. Regarding your first question, this is a retrospective study with all the biases that it implies. I would say that in our center, the most important thing is to achieve a complete repair, and all the patients are leaving the operating room with trivial MR. That being said, one could argue that when a patient presents with a heavy ischemic burden, one will be more inclined to repair the valve, compared with a patient who presents with an extremely dilated LV or lots of tethering, where surgeons might be more inclined to replace the valve. This is, however, certainly a good question, and it warrants prospective randomized studies to answer.

In regard to your second question, as you pointed out, lots of studies have shown that these patients are really sick, especially the subgroup with low ejection fractions. We don't have the incidence of ventricular assist device or heart transplantation in this population because this is a retrospective study. Furthermore, we don't have the incidence of late recurrence of MR or readmission for symptomatic heart failure.

However, in regard to ventricular assist device or heart transplantation, we are certainly looking at this population of sick patients, and we believe there might be a subgroup of patients with poor targets for bypasses who could be candidates for these advanced heart failure therapies even as a first option of treatment.

In regard to MR recurrence, although all those patients are leaving the operating room with trivial MR, we know the amount of recurrence of MR is approximately $20 \%$ to $30 \%$. This can influence a surgeon's decision to replace or repair the valve initially according to the patient's symptoms. If the patient presents with severe angina symptoms, the most important thing is to perform $\mathrm{CABG}$, but if the patient presents with long-term congestive heart failure symptoms, the most important thing is to have no recurrence of MR. This might influence the surgeon's decision.

Dr McCarthy. What would you do now?

Dr Maltais. This is a simple study that has shown us that when we look at the overall population, the type of procedure didn't seem to influence the overall survival. This study was intended to be simple, to look at what is really important in this population. From these results, the most important aspect seems to be patient selection. If the patient has an MVP, it has to be quick and efficient. Only the underlying patients' condition and presentation at the time of surgery seem to influence survival. 
Dr Ottavio Alfieri (Milan, Italy). May I ask which kind of repair you carried out?

Dr Maltais. This is it. A retrospective study, but more than $90 \%$ of those patients had an undersized ring. Patients with mixed disease are a little harder to analyze, but all those patients had IMR, and neochordae and leaflet resection were the most frequent concomitant techniques used.

Dr Robert Dion (Genk, Belgium). I would like to return to the technique. When you repair the valve, do you use a complete, pliable, or rigid ring? If you undersize, when and how much do you undersize?

Dr Maltais. With regard to the type of repair, the numbers are small when we looked at subgroups, but the type of ring did not change results. Most of the surgeons try to undersize 2 sizes.

Dr Dion. If you replace the valve, do you systematically use a bioprosthesis, and if you use a bioprosthesis, what sizing are you applying? Are you aiming at the largest possible size or trying to somewhat shrink the base of the heart by using some undersizing? What is the policy?

Dr Maltais. We don't have any policy to look at those results specifically. In patients with replacement, all had preservation of the posterior mitral apparatus, and we believe this is the most important thing.

Dr Paul Kurlansky (Miami, Fla). Very interesting and provocative work. As you probably know, there are emerging data on IMR that although the patients with MR have a worse prognosis, it doesn't necessarily seem to matter whether or not you repair/ replace the valve in terms of their long-term survival. So in view of that, I was just wondering what exactly are your indications for surgically addressing the MV?

Dr Maltais. Patients with moderate IMR are another topic. But these are all patients who needed to have a procedure performed on the MV, and therefore all patients had more than moderate MR. If repair is possible, this is the procedure of choice.

Dr Thierry Mesana (Ottawa, Ontario, Canada). A great study, Simon, and great results. We actually presented the same study with propensity case matching at the Society of Thoracic Surgeons. First, in your population with MVP, you had a high number of patients with redos, were these patients with previous MVR? Second, did you look at the LV function in both groups? Did you see a difference in recovery of LV function with one or the other group and eventually LV size changes after the LV repair versus replacement?

Dr Maltais. In terms of MVR, most of the patients with a previous MVP had MVR. This was a retrospective study, again, and the hard thing to point out is how many patients had replacement because they had 1 or 2 failed attempts to repair.

In terms of recurrence of MR and LV remodeling, this is something we are looking into. Approximately $20 \%$ to $30 \%$ of those patients will have recurrence of MR, which reflects the underlying disease and ventricular problem. In further studies, we will be looking into LV dimension reduction or recuperation of LV function as a potential marker of survival in patients with recurrent MR.

Dr Mesana. A great study, and actually we had presented similar results, including the recurrence of MR, which did not affect long-term survival.

Dr Maltais. Thank you.

Dr Steven Bolling (Ann Arbor, Mich). Simon, a very nice study. You don't have long-term echocardiography follow-up on these patients, is that correct?

Dr Maltais. We do have echocardiography follow-up on those patients but with midterm follow-up.

Dr Bolling. So then, I will ask you in a different way. Is it possible that on your curve you actually have 3 groups, good mitral repair, mitral replacement, and bad mitral repair, meaning a bunch of patients who had recurrence. Could it be you are not really looking at MVP versus MVR, but whether the patients have recurrent MR in one group and not in another and whether they were able to remodel their ventricles. Do you have enough data and follow-up echocardiography to say that?

Dr Maltais. At this point, I would say we don't. We are currently looking at those data and trying to assess this more in detail. The main advantage of MVR compared with MVP is obviously the fact that all those patients don't have recurrent MR. As you pointed out, there might be a subgroup of patients with recurrent MR with a higher mortality compared with patients with perfect repairs and MVR.

Dr Soon Park. I think we need to separate a few things out for discussion. The National Heart, Lung, and Blood Institute Cardiothoracic Surgical Trial Network study may inform us about what might happen to the LV geometry after repair versus replacement, but it probably will not tell us much about survival. The current study is a retrospective review, and its primary focus is on survival. We really do not know how recurrent MR might affect survival or the impact of MVR, whether it has a significant adverse impact or not. Clearly, we need to study these issues further in the future. Meanwhile, this study seems to illustrate important factors that influence the outcome after surgery in this high-risk group, and it seems that MVR versus MVP does not make a difference. 